\title{
Origin of Plasmodium falciparum malaria is traced by mitochondrial DNA
}

David J. Conwaya, a , $\square$, Caterina Fanelloa, Jennifer M. Lloyd ${ }^{a}$, Ban M.A.-S. Al-Jouboria, Aftab H. Baloch $^{a}$, Sushela D. Somanath ${ }^{a}$, Cally Roper ${ }^{a}$, Ayoade M.J. Oduola ${ }^{b}, B_{e r t ~ M u l d e r}$, Marinete M.

Povoa $^{d}$, Balbir Singhe, Alan W. Thomas ${ }^{\mathrm{f}}$

a Department of Infectious and Tropical Diseases, London School of Hygiene and Tropical Medicine, Keppel St, London WC1E 7HT, UK

b College of Medicine, University of Ibadan, Ibadan, Nigeria

c Malaria Department, OCEAC, Yaounde, Cameroon

d Servico de Parasitologia, Instituto Evandro Chagas, Belem PA, Brazil

e Universiti Malaysia Sarawak, Kota Samarahan 94300, Sarawak, Malaysia

f Biomedical Primate Research Centre, PO Box 3306, 2288 GH Rijswijk, The Netherlands

\begin{abstract}
The origin and geographical spread of Plasmodium falciparum is here determined by analysis of mitochondrial DNA sequence polymorphism and divergence from its most closely related species $P$. reichenowi (a rare parasite of chimpanzees). The complete $6 \mathrm{~kb}$ mitochondrial genome was sequenced from the single known isolate of $P$. reichenowi and from four different cultured isolates of $P$. falciparum, and aligned with the two previously derived $P$. falciparum sequences. The extremely low synonymousnucleotide polymorphism in $P$. falciparum $(n=0.0004)$ contrasts with the divergence at such sites between the two species $(K=0.1201)$, and supports a hypothesis that $P$. falciparum has recently emerged from a single ancestral population. To survey the geographical distribution of mitochondrial haplotypes in $P$. falciparum, 104 isolates from several endemic areas were typed for each of the identified single nucleotide polymorphisms. The haplotypes show a radiation out of Africa, with unique types in Southeast Asia and South America being related to African types by single nucleotide changes. This indicates that $P$. falciparum originated in Africa and colonised Southeast Asia and South America separately.
\end{abstract}

Keywords: Plasmodium falciparum; Plasmodium reichenowi; Mitochondrial genome; Genetics; Species; Evolution

Abbreviations: mt, mitochondrial; nt, nucleotide

Published in Molecular and Biochemical Parasitology, Volume 111, Issue 1, November 2000, Pages 163-171. 